Canad. Math. Bull. Vol. 21 (1), 1978

\title{
THIN LENS SPACES
}

\author{
P. HOFFMAN AND A. ZABRODSKY
}

In Theorem 1 below we study the existence of spaces whose cohomology rings are isomorphic (as ungraded rings) to those of lens spaces. The case $p=2$ is very simple and instructive, so let us consider it first.

Suppose $X$ is a space such that $H^{*}(X) \cong Z[x] /\left\langle 2 x, x^{3}\right\rangle$ where $\operatorname{dim} x=2 d$ (for example $X=R P^{4}$ with $d=1$ ). Then the Atiyah-Hirzebruch spectral sequence collapses, so $\tilde{K}(X)$ has an element $y$ such that either

(i) $\tilde{K}(X) \cong Z_{4}$ generated by $y$, and $y^{2}=2 y$, or

(ii) $\tilde{K}(X) \cong Z_{2} \oplus Z_{2}$ with generators $y$ and $y^{2}$.

But, in case (ii),

$$
0=\lambda^{2}(0)=\lambda^{2}(2 y)=2 \lambda^{2}(y)+y^{2}=y^{2}, \quad \text { a contradiction. }
$$

Hence we must have case (i). In general

$$
\lambda^{2}(a b)=a^{2} \lambda^{2}(b)+b^{2} \lambda^{2}(a)-2 \lambda^{2}(a) \lambda^{2}(b) .
$$

Thus, since $2 \tilde{K}(X) \cdot \tilde{K}(X)=0$,

$$
\begin{aligned}
2 y+2 \lambda^{2}(y) & =y^{2}+2 \lambda^{2}(y)=\lambda^{2}(2 y)=\lambda^{2}\left(y^{2}\right) \\
& =2 y^{2} \lambda^{2}(y)-2\left[\lambda^{2}(y)\right]^{2}=0 .
\end{aligned}
$$

Hence $\lambda^{2}(y) \equiv-y\left(\bmod y^{2}\right)$. But in general, if $y \in K_{2 d}$ the $d$ th filtration subgroup, then $\lambda^{2}(y) \equiv-2^{d-1} y\left(\bmod K_{2 d+2}\right)$. Thus we must have $d=1$, so $X$ looks much like $R P^{4}$.

The corresponding result for odd primes goes as follows.

THEOREM 1. (a) If $p$ is a prime and $d$ is a positive integer, then there is a space $X$ with $H^{*}(X) \cong Z[x] /\langle p x\rangle$ where $\operatorname{dim} x=2 d$ if and only if $d$ is a divisor of $p-1$.

(b) More precisely, $Z[x] /\left\langle p x, x^{p+1}\right\rangle$ is not realizable as an integral cohomology ring unless $\operatorname{dim} x / 2$ divides $p-1$.

The proof determines the group extensions in the computation of the $K$-theory of skeletons of $X$ for certain $d$. This includes $d=1$, where the lens spaces are examples. It gives a simple algebraic determination of their $K$ theory, normally done by exhibiting bundles, or better, representations of cyclic groups. ${ }^{(1)}$

Received by the editors May 18, 1977. tions.

(1) R. Kane has pointed out another proof of half of the theorem using cohomology opera- 
We both wish to thank the Oxford Mathematical Institute for its hospitality during the autumn of 1975 . We also thank the referee for several valuable suggestions, particularly the one described in the final paragraph.

A. Proof that $\boldsymbol{d}$ divides $\boldsymbol{p}-1$. The symmetric polynomial $p^{-1}\left[\sum x_{i}^{p}-\left(\sum x_{i}\right)^{p}\right]$ can be expressed as $f_{p}\left(\sigma_{1}, \ldots, \sigma_{p}\right)$, where $f_{p}$ is a polynomial with integer coefficients and $\sigma_{i}$ is the $i$ th elementary symmetric function. If $R$ is a special $\lambda$-ring [4], define $\Theta^{p}: R \rightarrow R$ as in [3]:

$$
\Theta^{p}(x)=f_{p}\left[\lambda^{1}(x), \ldots, \lambda^{p}(x)\right] .
$$

LEMMA 2. For $x \in R$ and positive integers $n$, we have

(a) $\Theta^{p}(n x)=n \Theta^{p}(x)+p^{-1}\left(n-n^{p}\right) x^{p}$.

(b) $\Theta^{p}\left(x^{n}\right)=\sum_{j=1}^{n} p^{j-1}\left(\begin{array}{l}n \\ j\end{array}\right) \Theta^{p}(x)^{j} x^{p(n-j)}$.

Proof. Since the free $\lambda$-ring on two generators is torsion free, these follow immediately from the properties of the Adams operations $\psi^{p}$ and the equation $\Theta^{p}(x)=p^{-1}\left[\psi^{p}(x)-x^{p}\right]$. Alternatively, they follow from the Verification Principle $[4 ; 3.2]$. Two more proofs are by induction on $n$, first working out the formulae for $\Theta^{p}(x+y)$ and $\Theta^{p}(x y)$ by either method above.

Note. There are two reasons why $\Theta^{p}$ is easier to use than $\lambda^{p}$ in this context: Theorem 3 below, and the fact that the formulae above involve sums, products and $\Theta^{p}$, but no other operations.

Theorem 3. (Atiyah) Suppose $Y$ is a finite complex with $H^{k}(Y)=0$ for all odd $k$. Then for $y \in K_{2 d}(Y)$, the image of $\Theta^{p}(y)$ in $K(Y) / K_{2 d+2 t+2}(Y)$ is divisible by $p^{d-[t / p-1]-1}$, where $[s]$ denotes the integer part of $s$.

This follows immediately from [3, Theorem 5.3].

In the remainder of section $A$, we assume $Y$ is a finite complex with $H^{*}(Y) \cong Z[x] /\left\langle p x, x^{p+1}\right\rangle$, where $\operatorname{dim} x=2 d$, and we prove $d$ divides $p-1$.

LEMMA 4. There exist $y \in \tilde{K}(Y)$ and integers $h$, with $2 \leq h \leq p+1$, and $a$, prime to $p$, such that

(i) $K(Y)=Z[y] /\left\langle p y-a y^{h}, y^{p+1}\right\rangle$;

(ii) $K_{2 t d}(X)$ is the group generated by $\left\{y^{i} \mid t \leq i \leq p\right\}$ for all $t$, and $K_{2 s}(Y)=K_{2 s+2}(Y)$ if $s+d$

(iii) $p-1$ is a divisor of $d(h-1)$, if $h \neq p+1$.

Proof. Since $H^{2 i+1}(Y)=0$ for all $i$, the Atiyah-Hirzebruch spectral sequence collapses. Thus we can find $y^{\prime}$ such that (ii) holds with $y^{\prime}$ in place of $y$ and such that

$$
K(Y)=Z\left[y^{\prime}\right] /\left\langle p y^{\prime}-\sum_{i=h}^{p} a_{i} y^{\prime i}, y^{\prime p+1}\right\rangle
$$


where $2 \leq h \leq p+1$ and $a_{h}$ is prime to $p$. By [1, Cor. 8], $\tilde{K}(Y)$ splits as a direct sum $G^{(0)} \oplus G^{(1)} \oplus \cdots \oplus G^{(p-2)}$ of filtered groups, where for each index $\alpha \epsilon$ $Z_{p-1}$, we have $G_{2 i}^{\alpha}=G_{2 i+2}^{\alpha}$ if $i \notin \alpha$. Since $y^{\prime} \in K_{2 d}(Y)$, by projecting into $G^{(d)}$, we may take $y^{\prime} \in G^{(d)}$ without affecting anything but the exact values of the coefficients $a_{i}$. To verify (iii), note that if $h \neq p+1$, we have

$$
p y^{\prime}=\sum_{i=h}^{p} a_{i} y^{i} \in G_{2 d h}^{(d)}-G_{2 d h+2}^{(d)} .
$$

Hence $d h \in(d)$ i.e. $d h \equiv d \bmod (p-1)$, proving (iii). Now, without affecting (ii), we can alter $y^{\prime}$ to make $a_{h+1}=0$ by setting $y^{\prime \prime}=y^{\prime}+a_{h+1} \bar{a}_{h}(\overline{h-1}) y^{\prime 2}$, where $\bar{c} c \equiv 1(\bmod p)$. Continuing inductively we can eliminate $a_{h+2}, \ldots, a_{p}$ arriving at an element $y$ such that (i) holds.

LEMMA 5. $d(h-1)^{2} \leq(p-1)^{2}$.

Proof. By (2a) and (b) we have

$$
\Theta^{p}(p y)=p \Theta^{p}(y)+y^{p}
$$

and

$$
\begin{aligned}
\Theta^{p}\left(a y^{h}\right) & =a \Theta^{p}\left(y^{h}\right)+\frac{a-a^{p}}{p} y^{p h}=a \Theta^{p}\left(y^{h}\right) \\
& =a \sum_{j=1}^{h} p^{j-1}\left(\begin{array}{l}
h \\
j
\end{array}\right)\left[\Theta^{p}(y)\right]^{j} y^{p(h-j)}=a p^{h-1}\left[\Theta^{p}(y)\right]^{h}
\end{aligned}
$$

since $y^{p(h-j)}=0$ unless $j=h$ or $h-1$, and since $\left[\Theta^{p}(y)\right]^{h-1} y^{p}=0$. Since $p y=$ $a y^{h}$, we obtain $p \Theta^{p}(y)+y^{p}=a p^{h-1}\left[\Theta^{p}(y)\right]^{h}$. Since $h \geq 2$, the filtration of $a p^{h-1}\left[\Theta^{p}(y)\right]^{h}$ is strictly larger than that of $p \Theta^{p}(y)$, a non-zero element since $y^{p} \neq 0$. But $y^{p}$ is in the last non-zero filtration subgroup, so we must have $p \Theta^{p}(y)+y^{p}=0$. This implies $p \tilde{K}(Y) \neq 0$ i.e. $h \neq p+1$, and that $\Theta^{p}(y) \equiv$ $b y^{p-h+1} \bmod K_{2 d(p-h+1)+2}(Y)$ for some $b$ prime to $p$. In $K(Y) / K_{2 d(p-h+1)+2}(Y)$, $y^{p-h+1}$ is divisible by exactly $p^{[(p-1) /(h-1)]-1}$. By Theorem 3 ,

$$
d-\left[\frac{d(p-h)}{p-1}\right]-1 \leq\left[\frac{p-1}{h-1}\right]-1
$$

But

$$
\frac{d(p-h)}{p-1}=d-\frac{d(h-1)}{p-1}
$$

is an integer by 4(iii), so we obtain

$$
\frac{d(h-1)}{p-1} \leq\left[\frac{p-1}{h-1}\right] \quad \text { or } \quad d \leq\left[\frac{p-1}{h-1}\right]\left(\frac{p-1}{h-1}\right) \leq\left(\frac{p-1}{h-1}\right)^{2},
$$

as required. 
Proof that $\boldsymbol{d} \mid \boldsymbol{p}-1:$ If $\bar{x} \in H^{2 d}\left(Y ; Z_{p}\right)$ is the reduction of $x$, then $\mathscr{P}^{d}(\bar{x})=$ $\bar{x}^{p} \neq 0$. The usual argument using $\mathscr{P}^{1} \mathscr{P}^{d-1}=d \mathscr{P}^{d}$ then shows that either $d \mid p-1$ or $p \mid d$. But, if $d=s p$, Lemma 5 gives $s p(h-1)^{2} \leq(p-1)^{2}$. Lemma (4) (iii) gives $p-1 \mid s p(h-1)$, so

$$
p-1 \mid s(h-1) \leq(p-1)^{2} p^{-1}(h-1)^{-1}<p-1 .
$$

This contradiction eliminates the possibility that $p \mid d$, and so $d \mid p-1$, as required.

NotE. It is immediate from 4 (iii) and 5 that $h=p$ if and only if $d=1$, giving the group extension for the $K$-theory of a lens space. W. M. Chan has made some calculations analogous to these for the case when $p$ is not prime. He also noticed a gap in an earlier version of the proof above.

B. Construction of $\mathbf{X}$. In [5], a method is given for realizing in certain cases the image of an induced map as the cohomology of a space. A simple case is the following. Induced maps $f^{*}, s^{*}, t^{*}$ and $q^{*}$ always refer to $Z_{p}$-cohomology.

THEOREM 2. Given $f: W \rightarrow Y$, assume

(a) $H^{k}(W)$ and $H^{k}(Y)$ are finite $p$-groups for all $k>0$, and $Y$ is at least 2-connected;

(b) there exist maps $s$ and $t$ such that

(i)

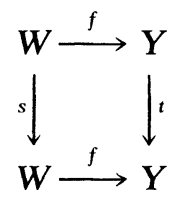

commutes,

(ii) $s^{*}$ and $t^{*}$ are diagonalizable,

(iii) the $\lambda$-eigenspace of $s^{*}$ lies in the image of $f^{*}$ for any product $\lambda$ of eigenvalues of $t^{*}$.

Then $f$ factors as $W \stackrel{g}{\longrightarrow} X \stackrel{\text { h }}{\longrightarrow} Y$ where

(1) $H^{k}(X)$ is a finite $p$-group for all $k>0$;

(2) $g^{*}$ is injective;

(3) $\operatorname{Im} g^{*}=\operatorname{Im} f^{*}$.

To construct $X$, a lens space will do for $d=1$, so we may take $d>1$ with $d \mid p-1$. Let $W=K\left(Z_{p}, 1\right)$ and $Y=K\left(Z_{p}, 2 d-1\right)$ and specify $f$ in Theorem 2 by $f^{*}\left(\iota_{2 d-1}\right)=\iota_{1}\left(\beta \iota_{1}\right)^{d-1}$. Here $\iota$ is the fundamental class for the EilenbergMaclane space and $\beta$ is the Bockstein operator. That $X$ has the required cohomology ring is elementary.

We are grateful to the referee for pointing out that such spaces $X$ occur "in nature" as well (see [2] for analogues): The field $Z_{p}$ has a multiplicative 
subgroup $S$ of order $d$. Let $G$ be the split extension of $Z_{p}$ by $S$, the action being multiplication. Let $\sigma: S \rightarrow G$ be a splitting and take $X=B_{G} v_{B \sigma} C B_{S}$. It follows that $H^{*}(X) \simeq \operatorname{ker} B_{\sigma}^{*}$. The action of $S$ on $H^{*}\left(B_{Z_{\mathrm{p}}}\right)$ as ring automorphisms may, on $H^{2}\left(B_{Z_{p}}\right) \cong Z_{p^{\prime}}$ be identified with the multiplication action. From the splitting

$$
H^{*}\left(B_{G}\right) \cong H^{*}\left(B_{Z_{p}}\right)^{s} \oplus H^{*}\left(B_{S}\right)
$$

it follows that $H^{*}(X) \cong H^{*}\left(B_{Z_{p}}\right)^{s}$, the fixed subring. This is what we want, since $S$ acts trivially exactly in the dimensions $2 k d$ for $k \geq 0$.

\title{
REFERENCES
}

1. J. F. Adams: Lecture 4, Splitting Generalized Cohomology Theories with coefficients, in Category Theory, Homology Theory and their Applications III, Springer Lecture Notes in Mathematics, Vol. 99.

2. J. F. Adams: The Kahn-Priddy Theorem, Proc. Comb. Phil. Soc. 73 (1973), 45-55.

3. M. F. Atiyah: Power Operations in K-theory, Quart. J. Math. Oxford (2), 17 (1966), 165-93.

4. M. F. Atiyah and D. O. Tall: Group Representations, $\lambda$-Rings and the J-Homomorphism, Topology 8 (1969), 253-97.

5. A. Zabrodsky: On the category of endomorphisms of finite complexes (to appear).

\author{
UNIVERSITY OF WATERLOO \\ Waterloo, Ontario, Canada \\ THE HeBReW UnIVERSITY \\ JERUSALEM
}

